\title{
Partial Volume Artifact
}

National Cancer Institute

\section{Source}

National Cancer Institute. Partial Volume Artifact. NCI Thesaurus. Code C87015.

A CT number underestimation artifact resulting from the presence of a dense object off center in a scan field. 